International Journal of Engineering \& Technology, $7(4.5)(2018) 254-256$
International Journal of Engineering \& Technology
SPC
Website: www.sciencepubco.com/index.php/IJET
Research paper

\title{
Design of a Circular Polarized Printed Rectenna for Satellite Solar Power Station Array Construction
}

\author{
Deepak Kumar ${ }^{1 *}$, Kalpana Chaudhary ${ }^{2}$ \\ ${ }^{I}$ Department of Electrical Engineering, Indian Institute of Technology (BHU) Varanasi 221005, India \\ ${ }^{2}$ Department of Electrical Engineering, Indian Institute of Technology (BHU) Varanasi 221005, India \\ *Corresponding author E-mail: deepak.rs.eee14@itbhu.ac.in
}

\begin{abstract}
A circularly polarized single feed microstrip patch antenna with voltage doubler rectification is designed at $2.45 \mathrm{GHz}$ for satellite solar wireless power transfer application. A bandpass filter is also designed and combined with an antenna that will efficiently eliminate signal harmonics up to third order. An HSMS-8202 microwave zero-bias Schottky barrier diodes accessible in SOT 23 package as the series pair is utilized in the proposed rectenna design. The rectenna has a high conversion efficiency of $70 \%$. The printed rectenna can be interconnected to construct the rectenna arrays.
\end{abstract}

Keywords: Antenna; Bandpass filter; circularly polarized; schottky diode; Rectenna.

\section{Introduction}

Remote power transmission is the transmission of electrical energy from a power source to an electrical gadget without power cables. It is mainly helpful in situations where interconnecting wires are difficult, insecure, or implausible [1]-[3]. Over the last decade, satellite solar power station (SSPS) and microwave wireless power transmission (WPT) have become an interesting topic for energy transmission in the future [4]. Since the execution of SSPS is fundamentally depended on the microwave production and transmission systems. Therefore, it requires microwave technology innovations on a large scale [5]. In a satellite solar power station, space satellite collects sun irradiance and photovoltaic transform it into electrical energy. This electrical power is then transformed into the microwave and transmitting that microwave power remotely to receiving antenna on Earth [5]. The receiving antenna connected rectifiers are used to convert microwave power back to electrical power. In the SSPS application, there is the demand for high DC output and to collect more RF energy a rectenna array is required [6], [7]. Also, the rectenna array that is capable of rectifying low microwave power is required. The rectenna array can be designed by utilizing different interconnections of rectenna components, and every association has its harvesting characteristic [8]. A few rectifier circuit investigation models have been proposed to analyze the rectenna performance [8]. It is not easy to develop an accurate model due to the nonlinear property of the diode [9]. It may be a linear circuit model or a nonlinear circuit model that can be utilized to predict the rectenna performance [9].

The rectenna is the combination of antenna and rectifier, and it is the most important part of the application of microwave wireless power transmission [4], [9]. The rectenna has been effectively utilized for a ground-to-ground demonstration of wireless power transmission. However, the technology advancement for space to ground power transmission is in the development stage. Wireless power transmission was explored and reviewed in [1]-[3]. A novel rectenna is reported in [7], it is made of a circularly polarized (CP), multi-band ring slot receiving antenna. It has been found useful in the application for versatile remote gadgets. In the other work, an antenna combined with a harmonic filter in the form of the circular sector is presented in [10]. The antenna with selffiltering capability can eliminate the use of the low-pass filter connection in between the antenna and rectifying diode. A rectangular patch microstrip antenna with a high gain is designed using coplanar waveguide (CPW) in [11]. The CPW rectenna configuration is very useful, and it's size reduced in comparison with two element microstrip patch antenna. In recent times, it has been shown that a CP antenna characteristic is essential for designing SSPS ground rectenna because in CP output voltage is not changed because of the revolution of the transmitter or receiver.

This work presents a CP printed rectenna circuit. The proposed rectenna in array form can be utilized in SSPS applications. This will provide a high opportunity for the flexible positioning of the rectenna cluster system. Therefore the losses due to the misalignment between transmitting antenna and receiving antennas can be reduced. Microstrip patch realization is suitable for compact rectenna design, and most important the printed technology will provide identical rectennas for efficient array development.

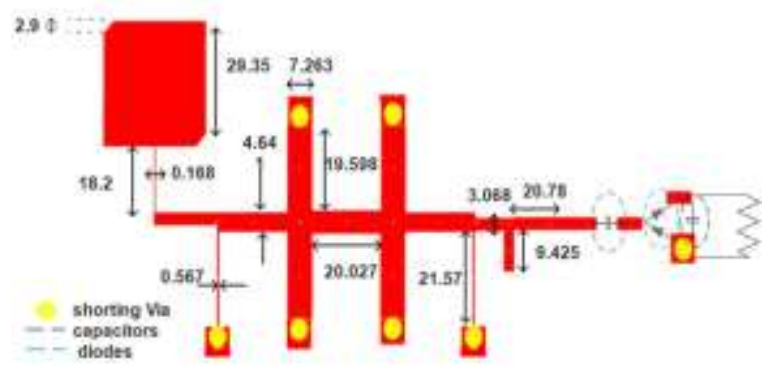

Fig. 1: A CP $2.45 \mathrm{GHz}$ voltage doubler rectenna design layout 


\section{Antenna with Band Pass Filter}

A circular polarized $(\mathrm{CP})$ truncated corner microstrip patch antenna is designed on FR4 substrate with dielectric constant (Er) 4.4, height $(h) 1.6 \mathrm{~mm}$, and a loss tangent of 0.02 ; the copper conductor thickness is $35 \mathrm{um}$. An EM full-wave 3-D simulator in ADS momentum is utilized to design the $\mathrm{CP}$ patch antenna and input bandpass filter (BPF) as shown in 'Fig. 1'. The antenna and BPF combined circuit measured return loss are shown in 'Fig. 2'. The measured return loss is $-14.23 \mathrm{~dB}$ at $2.45 \mathrm{GHz}$. The benefit of circular polarization is that the circuit rotation does not affect antenna performance. The patch antenna dimension and its mitered position requirement are precisely designed for good antenna performance, the dimensions have been shown in 'Fig. 1'. The truncated patch can provide the circular axial ratio (AR) below $3 \mathrm{~dB}$ [4]. The CP antenna has a gain of $5.012 \mathrm{~dB}$, and circular $\mathrm{AR}$ is $1.44 \mathrm{~dB}$ at $2.45 \mathrm{GHz}$. The CP Patch Antenna circular AR and gain with frequency variation have shown in 'Fig. 3'. The antenna is quarter wave matched with the 50-ohm transmission line. The CP antenna has layout coupling effect from BPF; subsequently, it will influence the antenna radiation pattern. Therefore, the $\mathrm{CP}$ antenna and BPF should be combined to measure antenna performance. The high performance grounded stubbed bandpass filter is designed to pass $2.45 \mathrm{GHz}$ of the signal from the antenna to the rectifier.

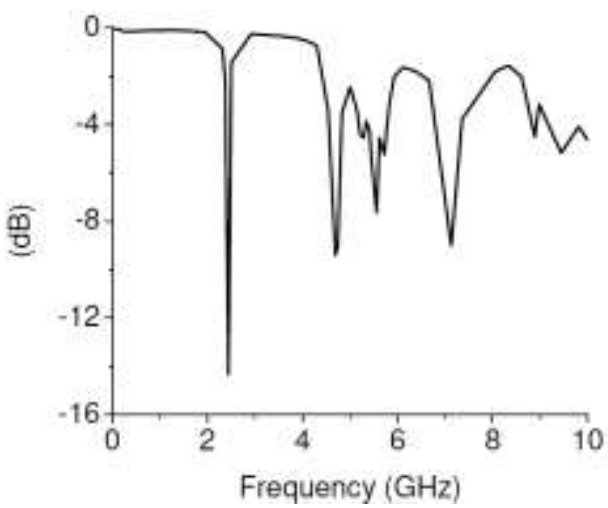

Fig. 2: Antenna with BPF measured return loss

\section{Rectifier Circuit and Impedance Matching}

Avago HSMS-8202 microwave Schottky diodes are utilized in the proposed rectenna design. These zero-bias Schottky barrier diodes are manufactured by Agilent Technologies and accessible in SOT 23 package as series pair configuration that is optimized for high-frequency operation (f $<10 \mathrm{GHz}$ ) [7]. This diode has Breakdown Voltage $\left(\mathrm{V}_{\mathrm{br}}\right)$ of $4 \mathrm{~V}$, forward voltage $\left(\mathrm{V}_{\mathrm{F}}\right)$ of $0.35 \mathrm{~V}$, junction capacitance $\left(\mathrm{C}_{\mathrm{j} 0}\right)$ of $0.18 \mathrm{pF}$, a series resistance $\left(\mathrm{R}_{\mathrm{s}}\right)$ of 6 $\Omega$ [7]. The above diode specifications are essential given the fact that RF to DC conversion efficiency of rectenna depends on these parameters. The rectifier diode in a voltage doubler setup is used in this design to obtain high voltage output.

A single stub microstrip matching is realized utilizing ADS Momentum to accomplish good impedance matching between the $\mathrm{RF}$ output and the rectifier's input. It will ensure maximum power transfer in connecting components and thus decrease the reflection loss at rectifier input. Initially, the rectifier configuration equivalent impedance is calculated for the desired frequency choice $(2.45$ $\mathrm{GHz}$ ) as demonstrated in 'Fig. 4'. As it has been observed that rectifier' input impedance is dependent on the received RF power at the antenna, therefore the matching is needed for predetermined input power.

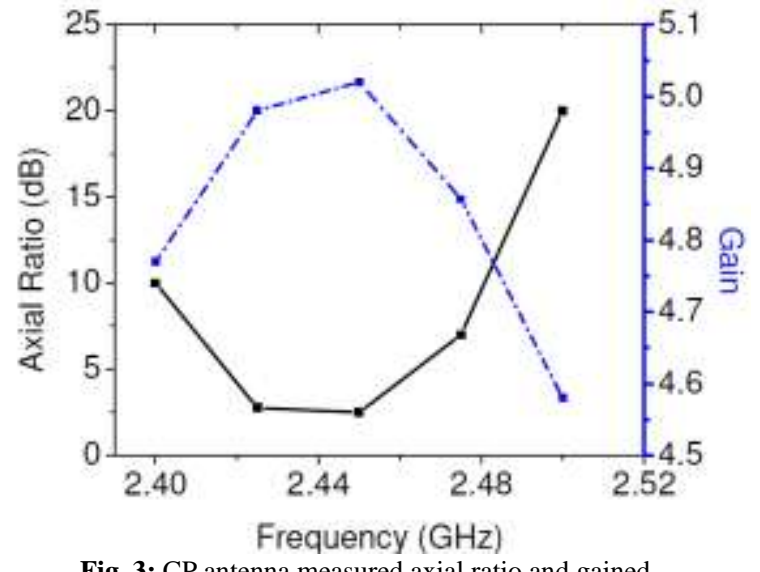

Fig. 3: $\mathrm{CP}$ antenna measured axial ratio and gained

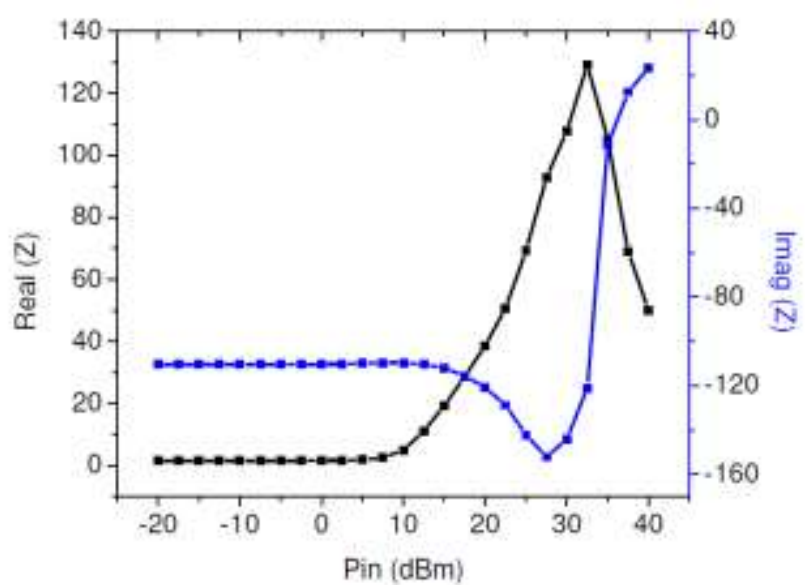

Fig. 4: Rectifier input impedance real and imaginary value variation with input power $(\mathrm{dBm})$

\section{Results and discussion}

The proposed rectenna is designed for a long range of power variation, that is $-10 \mathrm{dBm}$ to $30 \mathrm{dBm}$ at the receiving antenna. Accordingly, it is expected that $\mathrm{Pin}=10 \mathrm{dBm}$ is a good choice for the proposed rectifier matching network. A $2 \mathrm{~kW}$ output load is chosen in this design. A capacitor of $100 \mathrm{pf}$ is worked as an output filter. The rectifier diode in a voltage doubler setup is used in this design to obtain high voltage output. The measured value of rectenna output voltage and efficiency with varying input power density is plotted in 'Fig. 5' and 'Fig. 6' respectively. The designed rectenna has a maximum efficiency of $70 \%$ at ten $\mathrm{dBm}$ RF input power

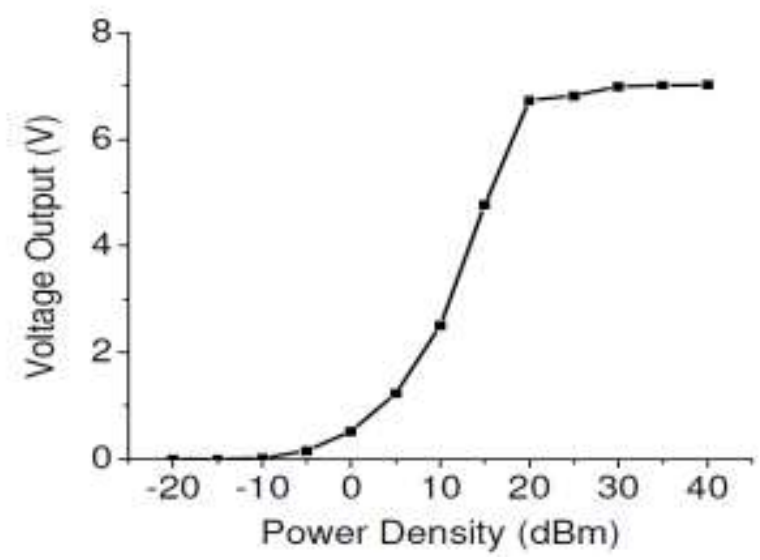

Fig. 5: Measured rectenna output voltage 


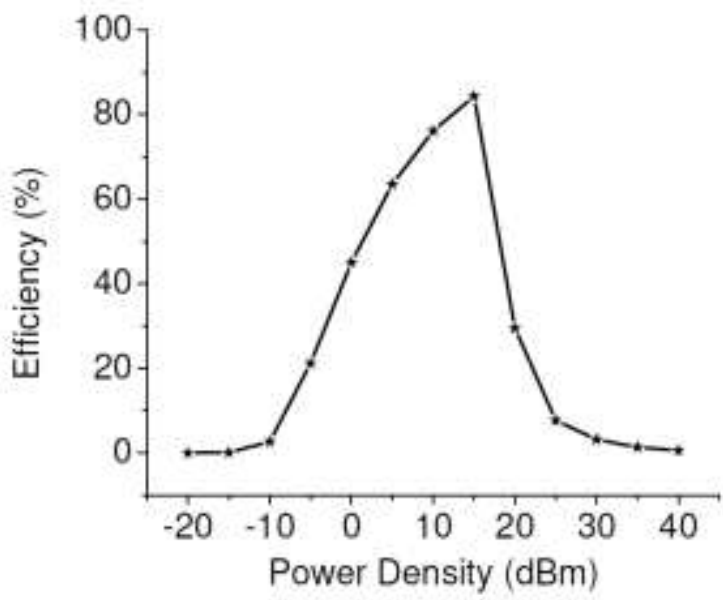

Fig. 6: Measured rectenna conversion efficiency

\section{Conclusions}

The gain and axial ratio of $5.012 \mathrm{~dB}$ and $1.44 \mathrm{~dB}$ respectively has been achieved by the designed truncated corner square patch antenna. Impedance matching is performed using Smith chart considering the ten $\mathrm{dBm}$ input power condition. The designed rectenna has a transformation efficiency of $70 \%$ that is independent of the positioning arrangement between the transmitting and receiving power antenna. The designed, printed rectenna is found suitable for SSPS application for microwave wireless power transfer.

\section{Acknowledgements}

We would like to thank all concerned with the Indian Institute of Technology (BHU) Varanasi for their all-out effort to support us in completing this research

\section{References}

[1] W. C. Brown, "Satellite Power Stations, A New Source of Energy," IEEE Spectrum, vol. 10, no. 3, pp. 38-47, 1973.

[2] W. C. Brown, "Beamed Microwave power transmission and its application to space," IEEE Trans. Microwave Theory Tech., vol. 40, no. 6 , p. 1239-1250., 1992

[3] W. C. Brown, "The History of Power Transmission by Radio Waves," IEEE Transactions on Microwave Theory and Techniques, vol. 32, pp. 1230-1242, 1984.

[4] D. Kumar and K. Chaudhary, "High-efficiency rectenna design for satellite solar power station," in 2016 IEEE Uttar Pradesh Section International Conference on Electrical, Computer and Electronics Engineering (UPCON), 2016, pp. 546-550.

[5] N. Shinohara and H. Matsumoto, "Microwave Power Transmission System with Phase and Amplitude Controlled Magnetrons," in Proceedings of 2nd International Conference on Recent Advances in Space Technologies (RAST 2005), 2004, pp. 28-33.

[6] J. Y. Siddiqui, S. Datta, M. Caillet, and Y. M. M. Antar, "Compact differentially fed inverted microstrip circular patch with an integrated coupler," IEEE Antennas and Wireless Propagation Letters, vol. 9, pp. 627-630, 2010 .

[7] H. Sun, Y. X. Guo, M. He, and Z. Zhong, "Design of a highefficiency 2.45-GHz rectenna for low-input-power energy harvesting," IEEE Antennas and Wireless Propagation Letters, vol. 11, pp. 929-932, 2012.

[8] D. Kumar and K. Chaudhary, "High Efficiency Harmonic Harvester Rectenna for Energy Storage Application,” International Journal of Power Electronics and Drive System (IJPEDS), vol. 9, no. 1, pp. 252-259, 2018.

[9] D. Kumar, "Analysis of satellite solar power station as base load power plant," 2016 IEEE Uttar Pradesh Section International Conference on Electrical, Computer and Electronics Engineering (UPCON), pp. 551-556, 2016.
[10] J. Y. Park, S.-M. Han, and T. Itoh, "A rectenna design with harmonic- rejecting circular-sector antenna," IEEE Antennas Wireless Propag. Lett., vol. 3, pp. 52-54, 2004.

[11] C. H. K. Chin, Q. Xue, and C. H. Chan, "Design of a 5.8-GHz rectenna incorporating a new patch antenna," IEEE Antennas and Wireless Propagation Letters, vol. 4, no. 1, pp. 175-178, 2005. 